\title{
Research Article \\ Dynamics Analysis of a Stochastic SIR Epidemic Model
}

\author{
Feng Rao \\ College of Sciences, Nanjing University of Technology, Nanjing 211816, China \\ Correspondence should be addressed to Feng Rao; raofeng2002@163.com
}

Received 2 January 2014; Accepted 27 January 2014; Published 2 March 2014

Academic Editor: Weiming Wang

Copyright (C) 2014 Feng Rao. This is an open access article distributed under the Creative Commons Attribution License, which permits unrestricted use, distribution, and reproduction in any medium, provided the original work is properly cited.

\begin{abstract}
We investigate an SIR epidemic model with stochastic perturbations. We assume that stochastic perturbations are of a white noise type which is directly proportional to the distances of three variables from the steady-state values, respectively. By constructing suitable Lyapunov functions and applying Itô's formula, some qualitative properties are obtained, such as the existence of global positive solutions, stochastic boundedness, and permanence. A series of numerical simulations to illustrate these mathematical findings are presented.
\end{abstract}

\section{Introduction}

Almost all mathematical models for the transmission of infectious diseases descend from the classical susceptibleinfective-removed (SIR) model of Kermack and McKendrick [1]. The dynamic behavior of different epidemic models and a lot of their extensions is well investigated by a number of scholars; see [2-11]. The basic and important research subjects for recent studies are the existence of the threshold values which distinguish whether the disease dies out, the stability of the disease-free and the endemic equilibria, permanence, and extinction [12]. During the last few decades, a number of realistic transmission functions have become the focus of considerable attention, and many authors are interested in the formulation of nonlinear incidence rate (see [13-17]). A nonlinear incidence rate can arise from saturation effects that if the proportion of the infection in a population is very high, so that exposure to the disease agent is virtually certain, then the transmission rate may respond more slowly than linear to the increase in the number of infection [18]. For example, Capasso and Serio [19] introduced a saturated transmission rate $f(S, I)=k S I /(1+\alpha I)$, where $k I$ measures the infection force of the disease and $1 /(1+\alpha I)$ measures the inhibition effect from the behavioral change of the susceptible individuals when their number increases or from the crowding effect of the infective individuals. To be biologically feasible, the function of the incidence rate $f(S, I)=k S I /(1+\alpha I)$ is a positive continuous and differentiable and satisfies the conditions

$$
\begin{gathered}
f(S, 0)=0=f(0, I), \\
\frac{\partial f(S, I)}{\partial S}=\frac{k I}{1+\alpha I}>0, \quad \frac{\partial f(S, I)}{\partial I}=\frac{k S}{(1+\alpha I)^{2}}>0,
\end{gathered}
$$

for all $S, I>0$. It is easy to know that the function $f(S, I)$ is concave with respect to the variable $I$; that is,

$$
\frac{\partial^{2} f(S, I)}{\partial I^{2}}=-\frac{2 \alpha k S}{(1+\alpha I)^{3}}<0 \quad \text { for } S, I>0,
$$

which implies that when the number of infections is very high that the exposure to the disease agent is virtually certain, the incidence rate will respond more slowly than linearly to the disease in $I$.

In the real world, population dynamics is inevitably subjected to environmental noise, which is an important component in an ecosystem. Most natural phenomena do not follow strictly deterministic laws but rather oscillate randomly about some average values, so that the population density never attains a fixed value with the advancement of time [20,21]. Recent advances in stochastic differential equations enable a lot of authors to introduce randomness into deterministic model of physical phenomena to reveal the effect of environmental variability, whether it is a random 
noise in the system of differential equations or environmental fluctuations in parameters; see [12, 13, 22-30]. Of them, Tuckwell and Williams [28] investigated the properties of a simple discrete time stochastic epidemic model. A classical model of an SIRS epidemic in an open population was considered by El Maroufy et al. [12]. They established the global stability of disease-free and endemic equilibrium points for both the deterministic and stochastic models. Based on the theory of stochastic differential equation, Cai et al. [13] studied the dynamics of an SIRS epidemic model with a ratiodependent incidence rate. In [29], the authors extended the classical SIRS epidemic model incorporating media coverage from a deterministic framework to a stochastic differential equation and focused on how environmental fluctuations of the contact coefficient affect the extinction of the disease.

To the best of our knowledge, a small amount of work has been done with stochastic perturbation on an SIR epidemic model with a saturated transmission rate $k S I /(1+\alpha I)$. The purpose of this paper is to study that the stochastic factor has a significant effect on the dynamics of SIR epidemic model with a saturated incidence rate. The organization of this paper is as follows. In the next section, we present the formulation of mathematical model with environmental noise. We give some properties about deterministic model (4) and carry out the analysis of the dynamical properties of stochastic model (3), respectively. Finally, we give a concluding section.

\section{Model and Dynamics Analysis}

Let $S(t)$ be the number of susceptible individuals, $I(t)$ the number of infective individuals, and $R(t)$ the number of removed individuals at time $t$, respectively. Motivated by [31], we assume that stochastic perturbations are of white noise type, which are directly proportional to distances $S(t), I(t), R(t)$ from the steady-state values of $S^{*}, I^{*}, R^{*}$ and influence on $\mathrm{d} S(t) / \mathrm{d} t, \mathrm{~d} I(t) / \mathrm{d} t, \mathrm{~d} R(t) / \mathrm{d} t$, respectively. In this way, an SIR epidemic model with a saturated transmission rate and stochastic fluctuations will be reduced to the following form:

$$
\begin{gathered}
\mathrm{d} S=\left(b-d S-\frac{k S I}{1+\alpha I}+\gamma R\right) \mathrm{d} t+\sigma_{1}\left(S-S^{*}\right) \mathrm{d} B(t), \\
\mathrm{d} I=\left(\frac{k S I}{1+\alpha I}-(d+\mu) I\right) \mathrm{d} t+\sigma_{2}\left(I-I^{*}\right) \mathrm{d} B(t) \\
\mathrm{d} R=(\mu I-(d+\gamma) R) \mathrm{d} t+\sigma_{3}\left(R-R^{*}\right) \mathrm{d} B(t) .
\end{gathered}
$$

All parameters are positive constants, $b$ is the recruitment rate of the population, $d$ is the natural death rate of the population, $k$ is the proportionality constant, $\alpha$ is the parameter that measures the psychological or inhibitory effect, $\gamma$ is the rate at which recovered individuals lose immunity and return to the susceptible class, and $\mu$ is the natural recovery rate of the infective individuals. Note that $\sigma_{1}, \sigma_{2}$, and $\sigma_{3}$ are real constants and known as the intensity of the stochastic environment and $B(t)$ is standard Brownian motion.
2.1. Dynamics of the Deterministic Model. In this subsection, when $\sigma_{1}=\sigma_{2}=\sigma_{3}=0$, we consider the deterministic SIR epidemic model:

$$
\begin{gathered}
\frac{\mathrm{d} S}{\mathrm{~d} t}=b-d S-\frac{k S I}{1+\alpha I}+\gamma R, \\
\frac{\mathrm{d} I}{\mathrm{~d} t}=\frac{k S I}{1+\alpha I}-(d+\mu) I, \\
\frac{\mathrm{d} R}{\mathrm{~d} t}=\mu I-(d+\gamma) R .
\end{gathered}
$$

Because of the biological meaning of the components $(S(t), I(t), R(t))$, we focus on the model in the first quadrant $\mathbb{R}_{+}^{3}=\left\{(S, I, R) \in \mathbb{R}^{3}: S \geq 0, I \geq 0, R \geq 0\right\}$. Model (4) always has a disease-free equilibrium $E_{0}=(b / d, 0,0)$, which corresponds to the extinction of the disease.

Define the basic reproduction number as

$$
R_{0}=\frac{b k}{d(d+\mu)},
$$

which denotes the number of individuals infected by a single infected individual placed in a totally susceptible population.

Theorem 1. From model (4), it follows that

(i) if $R_{0} \leq 1$, there is no positive equilibrium;

(ii) if $R_{0}>1$, there is a unique endemic equilibrium $E^{*}=$ $\left(S^{*}, I^{*}, R^{*}\right)$, which corresponds to the coexistence of $S$, $I$, and $R$ and is given by

$$
\begin{gathered}
S^{*}=\frac{(d+\mu)(d(d+\mu+\gamma)+\alpha b(d+\gamma))}{\alpha d(d+\mu)(d+\gamma)+d k(d+\mu+\gamma)}, \\
I^{*}=\frac{(d+\gamma)(b k-d(d+\mu))}{(d+\mu)(d(d+\mu+\gamma)+\alpha b(d+\gamma))} S^{*}, \\
R^{*}=\frac{\mu}{d+\gamma} I^{*} .
\end{gathered}
$$

In other words, when $R_{0}>1$, the disease can invade a totally susceptible population and the number of cases will increase, whereas when $R_{0} \leq 1$, the disease will always fail to spread.

Lemma 2. The plane $S+I+R=b / d$ is a manifold of model (4), which is attracting in the first octant.

Proof. Summing up the three equations in (4) and denoting $N(t)=S(t)+I(t)+R(t)$, we obtain

$$
\frac{\mathrm{d} N}{\mathrm{~d} t}=b-d N
$$

It is clear that $N(t)=b / d$ is a solution of (7) and for any $N\left(t_{0}\right) \geq 0$, the general solution of (7) is

$$
N(t)=\frac{1}{d}\left(b-\left(b-d N\left(t_{0}\right)\right) e^{-d\left(t-t_{0}\right)}\right) .
$$

Hence,

$$
\lim _{t \rightarrow \infty} N(t)=\frac{b}{d},
$$

which implies the conclusion. 
Theorem 3. The endemic equilibrium point $E^{*}=\left(S^{*}, I^{*}, R^{*}\right)$ is globally asymptotically stable in $\mathbb{R}_{+}^{3}$.

Proof. The Jacobian matrix $\mathbf{J}(S, I, R)$ at equilibrium point $E^{*}=\left(S^{*}, I^{*}, R^{*}\right)$ is given by

$$
\mathbf{J}\left(E^{*}\right)=\left(\begin{array}{lll}
J_{11} & J_{12} & J_{13} \\
J_{21} & J_{22} & J_{23} \\
J_{31} & J_{32} & J_{33}
\end{array}\right),
$$

where

$$
\begin{gathered}
J_{11}=-d-\frac{k I^{*}}{1+\alpha I^{*}}, \quad J_{12}=-\frac{k S^{*}}{\left(1+\alpha I^{*}\right)^{2}}, \quad J_{13}=\gamma, \\
J_{21}=\frac{k I^{*}}{1+\alpha I^{*}}, \quad J_{22}=\frac{k S^{*}}{\left(1+\alpha I^{*}\right)^{2}}-d-\mu, \quad J_{23}=0, \\
J_{31}=0, \quad J_{32}=\mu, \quad J_{33}=-d-\gamma .
\end{gathered}
$$

The characteristic equation at the interior equilibrium point $E^{*}$ is

$$
\lambda^{3}+Q_{1} \lambda^{2}+Q_{2} \lambda+Q_{3}=0
$$

where

$$
\begin{aligned}
& Q_{1}=-\left(J_{11}+J_{22}+J_{33}\right), \\
& Q_{2}=J_{11} J_{22}-J_{12} J_{21}+\left(J_{11}+J_{22}\right) J_{33}, \\
& Q_{3}=J_{12} J_{21} J_{33}-J_{21} J_{13} J_{32}-J_{11} J_{22} J_{33} .
\end{aligned}
$$

It is clear that

$$
\begin{gathered}
J_{11}<0, \quad J_{12}<0, \quad J_{13}>0, \quad J_{21}>0, \\
J_{22}<0, \quad J_{32}>0, \quad J_{33}<0 .
\end{gathered}
$$

Here $Q_{1}>0, Q_{2}>0$ and $Q_{3}>0$.

Now $Q_{1} Q_{2}-Q_{3}=\left(J_{11}+J_{22}\right) J_{12} J_{21}+J_{21} J_{13} J_{32}-J_{11}^{2}\left(J_{22}+\right.$ $\left.J_{33}\right)-J_{22}^{2}\left(J_{11}+J_{33}\right)-J_{33}^{2}\left(J_{11}+J_{22}\right)-2 J_{11} J_{22} J_{33}>0$. Therefore, model (4) is globally stable at the equilibrium $E^{*}=$ $\left(S^{*}, I^{*}, R^{*}\right)$.

2.2. Dynamics of the Stochastic Model. Let $(\Omega, \mathscr{F}, \mathbf{P})$ be a complete probability space with a filtration $\left\{\mathscr{F}_{t}\right\}_{t \in \mathbb{R}_{+}}$satisfying the usual conditions; that is, it is right continuous and increasing while $\mathscr{F}_{0}$ contains all P-null sets. Denote

$$
X(t)=(S(t), I(t), R(t)) \triangleq\left(x_{1}(t), x_{2}(t), x_{3}(t)\right),
$$

and the norm $|X(t)|=\sqrt{S^{2}(t)+I^{2}(t)+R^{2}(t)}$. And denote $C^{2,1}\left(\mathbb{R}^{3} \times(0, \infty) ; \mathbb{R}_{+}\right)$as the family of all nonnegative functions $V(X, t)$ defined on $\mathbb{R}^{3} \times(0, \infty)$ such that they are continuously twice differentiable in $X$ and once in $t$.

We define the differential operator $\mathbf{L}$ associated with three-dimensional stochastic differential equation

$$
\mathrm{d} X(t)=f(X, t) \mathrm{d} t+g(X, t) \mathrm{d} B(t),
$$

as

$$
\mathbf{L}=\frac{\partial}{\partial t}+\sum_{i=1}^{3} f_{i}(X, t) \frac{\partial}{\partial x_{i}}+\frac{1}{2} \sum_{i, j=1}^{3}\left(g^{T}(X, t) g(X, t)\right)_{i j} \frac{\partial^{2}}{\partial x_{i} \partial x_{j}},
$$

where

$$
\begin{gathered}
f=\left(\begin{array}{c}
b-d S-\frac{k S I}{1+\alpha I}+\gamma R \\
\frac{k S I}{1+\alpha I}-(d+\mu) I \\
\mu I-(d+\gamma) R
\end{array}\right), \\
g=\operatorname{diag}\left(\sigma_{1}\left(S-S^{*}\right), \sigma_{2}\left(I-I^{*}\right), \sigma_{3}\left(R-R^{*}\right)\right) .
\end{gathered}
$$

If $\mathbf{L}$ acts on a function $V \in C^{2,1}\left(\mathbb{R}^{3} \times(0, \infty) ; \mathbb{R}_{+}\right)$, then we denote

$$
\begin{aligned}
\mathbf{L} V(X, t)= & V_{t}(X, t)+V_{X}(X, t) f(X, t) \\
& +\frac{1}{2} \operatorname{trace}\left(g^{T}(X, t) V_{X X}(X, t) g(X, t)\right),
\end{aligned}
$$

where $T$ means transposition.

In this subsection, we first show the existence of a unique positive global solution of the stochastic model (3).

Theorem 4. For model (3) and any given initial value $(S(0)$, $I(0), R(0)) \in \mathbb{R}_{+}^{3}$, there is a unique solution $(S(t), I(t), R(t))$ on $t \geq 0$ and will remain in $\mathbb{R}_{+}^{3}$ with probability one.

Proof. Since the coefficients of model (3) satisfy the local Lipschitz condition, there is a unique local solution on $\left[0, \tau_{e}\right)$, where $\tau_{e}$ is the explosion time. Therefore, by Itô's formula, the unique local solution of model (3) is positive. Next, let us show that this solution is global; that is, $\tau_{e}=\infty$ a.s.

Let $n_{0}>0$ be sufficiently large for $S(0), I(0)$, and $R(0)$ lying with the interval $\left[1 / n_{0}, n_{0}\right]$. For each integer $n \geq n_{0}$, define a sequence of stopping times by

$$
\begin{aligned}
\tau_{n}=\inf \{t & \in\left[0, \tau_{e}\right]: S(t) \notin\left(\frac{1}{n}, n\right) \\
& \text { or } \left.I(t) \notin\left(\frac{1}{n}, n\right) \text { or } R(t) \notin\left(\frac{1}{n}, n\right)\right\},
\end{aligned}
$$

where we set $\inf \emptyset=\infty$ ( $\emptyset$ represents the empty set) in this paper. Since $\tau_{n}$ is nondecreasing as $n \rightarrow \infty$, there exists the limit

$$
\tau_{\infty}=\lim _{n \rightarrow \infty} \tau_{n} .
$$

Then $\tau_{\infty} \leq \tau_{e}$ a.s. Now, we need to show $\tau_{\infty}=\infty$ a.s. If this statement is violated, then there exist $T>0$ and $\varepsilon \in(0,1)$ such that

$$
\mathbf{P}\left\{\tau_{\infty} \leq T\right\}>\varepsilon .
$$

Thus, there is an integer $n_{1} \geq n_{0}$ such that

$$
\mathbf{P}\left\{\tau_{n} \leq T\right\} \geq \varepsilon \quad \forall n \geq n_{1} .
$$


Define a $C^{3}$-function $V: \mathbb{R}_{+}^{3} \rightarrow \mathbb{R}_{+}$by

$V(S, I, R)=(S-1-\ln S)+(I-1-\ln I)+(R-1-\ln R)$,

which is a nonnegative function. If $(S(t), I(t), R(t)) \in \mathbb{R}_{+}^{3}$, by using Itô's formula, we compute

$$
\begin{aligned}
& \mathrm{d} V(S, I, R)=\left(\left(1-\frac{1}{S}\right)\left(b-d S-\frac{k S I}{1+\alpha I}+\gamma R\right)\right. \\
& +\left(1-\frac{1}{I}\right)\left(\frac{k S I}{1+\alpha I}-(d+\mu) I\right) \\
& +\left(1-\frac{1}{R}\right)(\mu I-(d+\gamma) R)+\frac{\sigma_{1}^{2}}{2}\left(1-\frac{S^{*}}{S}\right)^{2} \\
& \left.+\frac{\sigma_{2}^{2}}{2}\left(1-\frac{I^{*}}{I}\right)^{2}+\frac{\sigma_{3}^{2}}{2}\left(1-\frac{R^{*}}{R}\right)^{2}\right) \mathrm{d} t \\
& +\left(\sigma_{1}\left(1-\frac{1}{S}\right)\left(S-S^{*}\right)+\sigma_{2}\left(1-\frac{1}{I}\right)\left(I-I^{*}\right)\right. \\
& \left.+\sigma_{3}\left(1-\frac{1}{R}\right)\left(R-R^{*}\right)\right) \mathrm{d} B(t) \\
& =\left(b+3 d+\mu+\gamma+\frac{\sigma_{1}^{2}}{2}\left(1-\frac{S^{*}}{S}\right)^{2}\right. \\
& +\frac{\sigma_{2}^{2}}{2}\left(1-\frac{I^{*}}{I}\right)^{2}+\frac{\sigma_{3}^{2}}{2}\left(1-\frac{R^{*}}{R}\right)^{2} \\
& +\frac{k I}{1+\alpha I}-\frac{k S}{1+\alpha I}-d(S+I+R) \\
& \left.-\frac{b}{S}-\frac{\gamma R}{S}-\frac{\mu I}{R}\right) \mathrm{d} t \\
& +\left(\sigma_{1}\left(1-\frac{1}{S}\right)\left(S-S^{*}\right)+\sigma_{2}\left(1-\frac{1}{I}\right)\left(I-I^{*}\right)\right. \\
& \left.+\sigma_{3}\left(1-\frac{1}{R}\right)\left(R-R^{*}\right)\right) \mathrm{d} B(t) \\
& \leq\left(b+3 d+\mu+\gamma+\frac{\sigma_{1}^{2}}{2}\left(1-\frac{S^{*}}{S}\right)^{2}\right. \\
& \left.+\frac{\sigma_{2}^{2}}{2}\left(1-\frac{I^{*}}{I}\right)^{2}+\frac{\sigma_{3}^{2}}{2}\left(1-\frac{R^{*}}{R}\right)^{2}+\frac{k}{\alpha}\right) \mathrm{d} t \\
& +\left(\sigma_{1}\left(1-\frac{1}{S}\right)\left(S-S^{*}\right)+\sigma_{2}\left(1-\frac{1}{I}\right)\left(I-I^{*}\right)\right. \\
& \left.+\sigma_{3}\left(1-\frac{1}{R}\right)\left(R-R^{*}\right)\right) \mathrm{d} B(t)
\end{aligned}
$$

$\leq M \mathrm{~d} t$

$$
\begin{aligned}
& +\left(\sigma_{1}\left(1-\frac{1}{S}\right)\left(S-S^{*}\right)+\sigma_{2}\left(1-\frac{1}{I}\right)\left(I-I^{*}\right)\right. \\
& \left.+\sigma_{3}\left(1-\frac{1}{R}\right)\left(R-R^{*}\right)\right) \mathrm{d} B(t)
\end{aligned}
$$

where $M$ is a positive constant. Integrating both sides of the above inequality from 0 to $\tau_{n} \wedge T$, we get

$$
\begin{aligned}
& \int_{0}^{\tau_{n} \wedge T} \mathrm{~d} V(S(s), I(s), R(s)) \\
& \leq \int_{0}^{\tau_{n} \wedge T} M \mathrm{~d} s \\
& \quad+\int_{0}^{\tau_{n} \wedge T}\left(\sigma_{1}\left(1-\frac{1}{S}\right)\left(S-S^{*}\right)+\sigma_{2}\left(1-\frac{1}{I}\right)\left(I-I^{*}\right)\right. \\
& \left.\quad+\sigma_{3}\left(1-\frac{1}{R}\right)\left(R-R^{*}\right)\right) \mathrm{d} B(s),
\end{aligned}
$$

where $\tau_{n} \wedge T=\min \left\{\tau_{n}, T\right\}$. Then taking the expectations leads to

$$
\begin{gathered}
\mathrm{E} V\left(S\left(\tau_{n} \wedge T\right), I\left(\tau_{n} \wedge T\right), R\left(\tau_{n} \wedge T\right)\right) \\
\quad \leq V(S(0), I(0), R(0))+M T .
\end{gathered}
$$

Set $\Omega_{n}=\left\{\tau_{n} \leq T\right\}$ for $n \geq n_{1}$ and from (23), we have $\mathbf{P}\left(\Omega_{n}\right) \geq \varepsilon$. For every $v \in \Omega_{n}$, there are some $i$ such that $x_{i}\left(\tau_{n}, v\right)$ equals either $n$ or $1 / n$ for $i=1,2,3$; hence $V\left(S\left(\tau_{n}, v\right), I\left(\tau_{n}, v\right), R\left(\tau_{n}, v\right)\right)$ is no less than $\min \{n-1-$ $\ln n, 1 / n-1-\ln (1 / n)\}$. Then we obtain

$$
\begin{aligned}
& V(S(0), I(0), R(0))+M T \\
& \quad \geq \mathbf{E}\left(1_{\Omega_{n}(v)} V\left(S\left(\tau_{n}\right), I\left(\tau_{n}\right), R\left(\tau_{n}\right)\right)\right) \\
& \quad \geq \varepsilon \min \left\{n-1-\ln n, \frac{1}{n}-1-\ln \frac{1}{n}\right\},
\end{aligned}
$$

where $1_{\Omega_{n}(v)}$ is the indicator function of $\Omega_{n}$. Letting $n \rightarrow \infty$ leads to the contradiction $\infty=V(S(0), I(0), R(0))+M T<$ $\infty$. This completes the proof.

Theorem 4 shows that the solution to model (3) will remain in $\mathbb{R}_{+}^{3}$. The property makes us continue to discuss how the solution varies in $\mathbb{R}_{+}^{3}$ in more detail. Here, we present that the definition of stochastic ultimate boundedness [32] is one of the important topics in population dynamics and is defined as follows.

Definition 5. The solutions $X(t)=(S(t), I(t), R(t))$ of model (3) are said to be stochastically ultimately bounded, if for any $\varepsilon \in(0,1)$, there is a positive constant $\delta=\delta(\varepsilon)$, such that for any initial value $(S(0), I(0), R(0)) \in \mathbb{R}_{+}^{3}$, the solution $X(t)$ to model (3) has the property that

$$
\limsup _{t \rightarrow \infty} \mathbf{P}\{|X(t)|>\delta\}<\varepsilon .
$$


Theorem 6. The solutions of model (3) are stochastically ultimately bounded for any initial value $(S(0), I(0), R(0)) \in$ $\mathbb{R}_{+}^{3}$.

Proof. From Theorem 4 , the solution will remain in $\mathbb{R}_{+}^{3}$ for all $t \geq 0$ almost surely. Define a function

$$
V(S, I, R)=e^{t}\left(S^{\theta}+I^{\theta}+R^{\theta}\right),
$$

for $(S, I, R) \in \mathbb{R}_{+}^{3}$ and $\theta>1$. By Itô's formula we obtain

$$
\begin{gathered}
\mathrm{d} V(S, I, R) \\
=e^{t}\left(\theta S^{\theta-1}\left(b-d S-\frac{k S I}{1+\alpha I}+\gamma R\right)\right. \\
+\theta I^{\theta-1}\left(\frac{k S I}{1+\alpha I}-(d+\mu) I\right) \\
+\theta R^{\theta-1}(\mu I-(d+\gamma) R) \\
+\frac{\theta(\theta-1)}{2}\left(\sigma_{1}^{2} S^{\theta}\left(1-\frac{S^{*}}{S}\right)^{2}+\sigma_{2}^{2} I^{\theta}\left(1-\frac{I^{*}}{I}\right)^{2}\right. \\
+e^{t} \theta\left(\sigma_{1} S^{\theta}\left(1-\frac{S^{*}}{S}\right)+\sigma_{2} I^{\theta}\left(1-\frac{I^{*}}{I}\right)\right. \\
\left.+\sigma_{3} R^{\theta}\left(1-\frac{R^{*}}{R}\right)\right) \mathrm{d} B(t) \\
\left.+\sigma_{3} R^{\theta}\left(1-\frac{R^{*}}{R}\right)\right) \mathrm{d} B(t) \\
\leq e^{t} \mathrm{~d} t+e^{t} \theta\left(\sigma_{1} S^{\theta}\left(1-\frac{S^{*}}{S}\right)+\sigma_{2} I^{\theta}\left(1-\frac{I^{*}}{I}\right)\right.
\end{gathered}
$$

where $C>0$ is a suitable constant.

Based on Theorem 4 and from (31), we have

$$
\begin{gathered}
\mathbf{E}\left(e^{t \wedge \tau_{n}} V\left(S\left(t \wedge \tau_{n}\right), I\left(t \wedge \tau_{n}\right), R\left(t \wedge \tau_{n}\right)\right)\right) \\
\quad \leq V(S(0), I(0), R(0))+C \mathbf{E} \int_{0}^{t \wedge \tau_{n}} e^{s} \mathrm{~d} s .
\end{gathered}
$$

Letting $n \rightarrow \infty$ yields

$e^{t} \mathbf{E} V(S(t), I(t), R(t)) \leq V(S(0), I(0), R(0))+C\left(e^{t}-1\right)$,

which implies

$\mathbf{E} V(S(t), I(t), R(t)) \leq e^{-t} V(S(0), I(0), R(0))+C$.

Note that

$$
\begin{aligned}
|X(t)|^{\theta} & =\left(S^{2}(t)+I^{2}(t)+R^{2}(t)\right)^{\theta / 2} \\
& \leq 3^{\theta / 2} \max \left\{S^{\theta}(t), I^{\theta}(t), R^{\theta}(t)\right\} \\
& \leq 3^{\theta / 2}\left(S^{\theta}+I^{\theta}+R^{\theta}\right) .
\end{aligned}
$$

Then we get

$$
\mathbf{E}|X(t)|^{\theta} \leq 3^{\theta / 2}\left(e^{-t} V(S(0), I(0), R(0))+C\right),
$$

which means

$$
\limsup _{t \rightarrow \infty} \mathbf{E}|X(t)|^{\theta} \leq 3^{\theta / 2} C<\infty
$$

Therefore, there exists a positive constant $\delta_{1}$ such that

$$
\limsup _{t \rightarrow \infty} \mathbf{E}|\sqrt{X(t)}|<\delta_{1} \text {. }
$$

For any $\varepsilon>0$, set $\delta=\delta_{1}^{2} / \varepsilon^{2}$, then by Chebyshev's inequality,

$$
\mathbf{P}\{|X(t)>\delta|\} \leq \frac{\mathbf{E}|\sqrt{X(t)}|}{\sqrt{\delta}} .
$$

Thus, we obtain

$$
\limsup _{t \rightarrow \infty} \mathbf{P}\{|X(t)>\delta|\} \leq \frac{\delta_{1}}{\sqrt{\delta}}=\varepsilon,
$$

which yields the required assertion.

Generally speaking, the nonexplosion property, the existence, and the uniqueness of the solution are not enough but the property of permanence is more desirable since it means the long time survival in a population dynamics. Now, the definition of stochastic permanence [33] will be given below.

Definition 7. The solutions $X(t)=(S(t), I(t), R(t))$ of model (3) are said to be stochastically permanent, if for any $\varepsilon \in(0,1)$, there exists a pair of positive constants $\delta=\delta(\varepsilon)$ and $\chi=\chi(\varepsilon)$ such that for any initial value $(S(0), I(0), R(0)) \in \mathbb{R}_{+}^{3}$, the solution $X(t)$ to model (3) has the properties

$$
\begin{aligned}
& \liminf _{t \rightarrow \infty} \mathbf{P}\{|X(t)| \leq \delta\} \geq 1-\varepsilon, \\
& \liminf _{t \rightarrow \infty} \mathbf{P}\{|X(t)| \geq \chi\} \geq 1-\varepsilon .
\end{aligned}
$$

Theorem 8. Assume $d<b$ and for any initial value $(S(0), I(0), R(0)) \in \mathbb{R}_{+}^{3}$, the solution $(S(t), I(t), R(t))$ satisfies

$$
\limsup _{t \rightarrow \infty} \mathbf{E}\left(|X(t)|^{-9}\right) \leq Q
$$

where $\vartheta$ is an arbitrary positive constant satisfying

$$
\frac{\vartheta+1}{2} \max \left\{\sigma_{1}^{2}, \sigma_{2}^{2}, \sigma_{3}^{2}\right\}<b-d
$$

$$
Q=\frac{3^{\vartheta}\left(4 \omega C_{1}+C_{2}\right)}{4 \omega C_{1}}
$$

$$
\times \max \left\{1,\left(\frac{2 C_{1}+C_{2}+\sqrt{C_{2}^{2}+4 C_{1} C_{2}}}{2 C_{1}}\right)^{9-2}\right\} \text {, }
$$


in which $\omega$ is an arbitrary positive constant satisfying

$$
\begin{gathered}
\omega<b-d-\frac{\vartheta+1}{2} \max \left\{\sigma_{1}^{2}, \sigma_{2}^{2}, \sigma_{3}^{2}\right\}, \\
C_{1}=b-d-\frac{\vartheta+1}{2} \max \left\{\sigma_{1}^{2}, \sigma_{2}^{2}, \sigma_{3}^{2}\right\}-\omega, \\
C_{2}=d+\max \left\{\sigma_{1}^{2}, \sigma_{2}^{2}, \sigma_{3}^{2}\right\}+2 \omega .
\end{gathered}
$$

Proof. Define a function

$$
V(S, I, R)=\frac{1}{S+I+R}
$$

for $(S(t), I(t), R(t)) \in \mathbb{R}_{+}^{3}$; using Itô's formula, we get

$\mathrm{d} V(S, I, R)$

$$
\begin{aligned}
= & -V^{2}(b-d(S+I+R)) \mathrm{d} t \\
& +V^{3}\left(\sigma_{1}^{2}\left(S-S^{*}\right)^{2}+\sigma_{2}^{2}\left(I-I^{*}\right)^{2}+\sigma_{3}^{2}\left(R-R^{*}\right)^{2}\right) \mathrm{d} t \\
& -V^{2}\left(\sigma_{1}\left(S-S^{*}\right)+\sigma_{2}\left(I-I^{*}\right)+\sigma_{3}\left(R-R^{*}\right)\right) \mathrm{d} B(t) .
\end{aligned}
$$

Choosing a positive constant $\vartheta$ that satisfies (43) and applying Itô's formula, we obtain

$$
\begin{aligned}
\mathbf{L}(1+V)^{9} \\
= \\
\qquad(1+V)^{\vartheta-1} \\
\times\left(-V^{2}(b-d(S+I+R))\right. \\
\left.\quad+V^{3}\left(\sigma_{1}^{2}\left(S-S^{*}\right)^{2}+\sigma_{2}^{2}\left(I-I^{*}\right)^{2}+\sigma_{3}^{2}\left(R-R^{*}\right)^{2}\right)\right) \\
+\frac{\vartheta(\vartheta-1)}{2} V^{4}(1+V)^{9-2} \\
\times\left(\sigma_{1}^{2}\left(S-S^{*}\right)^{2}+\sigma_{2}^{2}\left(I-I^{*}\right)^{2}+\sigma_{3}^{2}\left(R-R^{*}\right)^{2}\right) \\
=9(1+V)^{9-2} \\
\times\left(-V^{2}(b-d(S+I+R))-V^{3}(b-d(S+I+R))\right. \\
\quad+V^{3}\left(\sigma_{1}^{2}\left(S-S^{*}\right)^{2}+\sigma_{2}^{2}\left(I-I^{*}\right)^{2}+\sigma_{3}^{2}\left(R-R^{*}\right)^{2}\right) \\
\left.\quad+\frac{\vartheta+1}{2} V^{4}\left(\sigma_{1}^{2}\left(S-S^{*}\right)^{2}+\sigma_{2}^{2}\left(I-I^{*}\right)^{2}+\sigma_{3}^{2}\left(R-R^{*}\right)^{2}\right)\right) \\
=9(1+V)^{9-2} W,
\end{aligned}
$$

where

$$
\begin{aligned}
W= & -V^{2}(b-d(S+I+R))-V^{3}(b-d(S+I+R)) \\
& +V^{3}\left(\sigma_{1}^{2}\left(S-S^{*}\right)^{2}+\sigma_{2}^{2}\left(I-I^{*}\right)^{2}+\sigma_{3}^{2}\left(R-R^{*}\right)^{2}\right) \\
& +\frac{\vartheta+1}{2} V^{4}\left(\sigma_{1}^{2}\left(S-S^{*}\right)^{2}+\sigma_{2}^{2}\left(I-I^{*}\right)^{2}+\sigma_{3}^{2}\left(R-R^{*}\right)^{2}\right) \\
\leq & d V-(b-d) V^{2} \\
& +V^{3}\left(\sigma_{1}^{2}\left(S-S^{*}\right)^{2}+\sigma_{2}^{2}\left(I-I^{*}\right)^{2}+\sigma_{3}^{2}\left(R-R^{*}\right)^{2}\right) \\
& +\frac{\vartheta+1}{2} V^{4}\left(\sigma_{1}^{2}\left(S-S^{*}\right)^{2}+\sigma_{2}^{2}\left(I-I^{*}\right)^{2}+\sigma_{3}^{2}\left(R-R^{*}\right)^{2}\right) .
\end{aligned}
$$

Using the facts that

$$
\begin{aligned}
V^{3} & \left(\sigma_{1}^{2}\left(S-S^{*}\right)^{2}+\sigma_{2}^{2}\left(I-I^{*}\right)^{2}+\sigma_{3}^{2}\left(R-R^{*}\right)^{2}\right) \\
& <\max \left\{\sigma_{1}^{2}, \sigma_{2}^{2}, \sigma_{3}^{2}\right\} V, \\
V^{4} & \left(\sigma_{1}^{2}\left(S-S^{*}\right)^{2}+\sigma_{2}^{2}\left(I-I^{*}\right)^{2}+\sigma_{3}^{2}\left(R-R^{*}\right)^{2}\right) \\
& <\max \left\{\sigma_{1}^{2}, \sigma_{2}^{2}, \sigma_{3}^{2}\right\} V^{2},
\end{aligned}
$$

then,

$$
\begin{aligned}
W \leq & \left(d+\max \left\{\sigma_{1}^{2}, \sigma_{2}^{2}, \sigma_{3}^{2}\right\}\right) V \\
& -\left(b-d-\frac{\vartheta+1}{2} \max \left\{\sigma_{1}^{2}, \sigma_{2}^{2}, \sigma_{3}^{2}\right\}\right) V^{2} .
\end{aligned}
$$

Let $\omega>0$ be sufficiently small such that it satisfies (45), by Itô's formula; then

$$
\begin{aligned}
\mathbf{L}\left(e^{\omega t}(1+V)^{\vartheta}\right) & =\omega e^{\omega t}(1+V)^{\vartheta}+e^{\omega t} \mathbf{L}(1+V)^{\vartheta} \\
& =e^{\omega t}(1+V)^{\vartheta-2}\left(\omega(1+V)^{2}+W\right) \\
& \leq e^{\omega t}(1+V)^{\vartheta-2}\left(\omega-C_{1} V^{2}+C_{2} V\right) \\
& \leq Q_{0} e^{\omega t}
\end{aligned}
$$

where $Q_{0}=\left(4 \omega C_{1}+C_{2}\right) /\left(4 C_{1}\right) \max \left\{1,\left(\left(2 C_{1}+C_{2}+\right.\right.\right.$ $\left.\left.\left.\sqrt{C_{2}^{2}+4 C_{1} C_{2}}\right) /\left(2 C_{1}\right)\right)^{9-2}\right\}$ and $C_{1}, C_{2}$ have been defined in the statement of the theorem. Thus,

$$
\mathbf{E}\left(e^{\omega t}(1+V)^{\vartheta}\right) \leq(1+V(0))^{\vartheta}+\frac{Q_{0}}{\omega} e^{\omega t} .
$$

Therefore we obtain

$$
\limsup _{t \rightarrow \infty} \mathbf{E}\left(V(t)^{\vartheta}\right) \leq \limsup _{t \rightarrow \infty} \mathbf{E}(1+V)^{\vartheta} \leq \frac{Q_{0}}{\omega} .
$$

For $(S, I, R) \in \mathbb{R}_{+}^{3}$, we know that $(S+I+R)^{\vartheta} \leq 3^{\vartheta}\left(S^{2}+\right.$ $\left.I^{2}+R^{2}\right)^{\vartheta / 2} \leq 3^{\vartheta}|X(t)|^{\vartheta}$; consequently,

$$
\limsup _{t \rightarrow \infty} \mathbf{E}\left(\frac{1}{|X(t)|^{\vartheta}}\right) \leq 3^{\vartheta} \limsup _{t \rightarrow \infty} \mathbf{E}\left(V(t)^{\vartheta}\right) \leq \frac{3^{\vartheta} Q_{0}}{\omega}=Q,
$$

which completes the proof. 


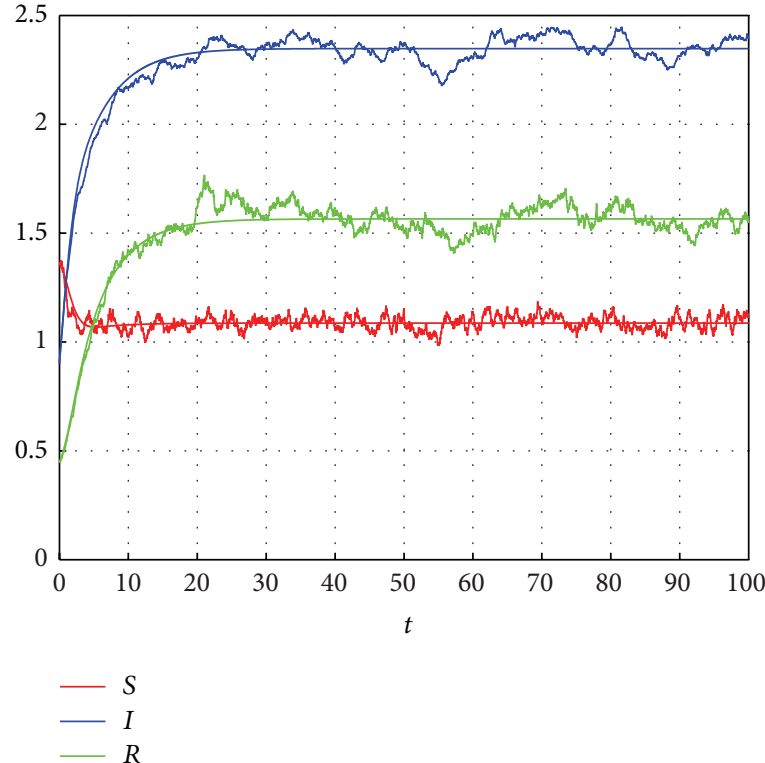

(a)

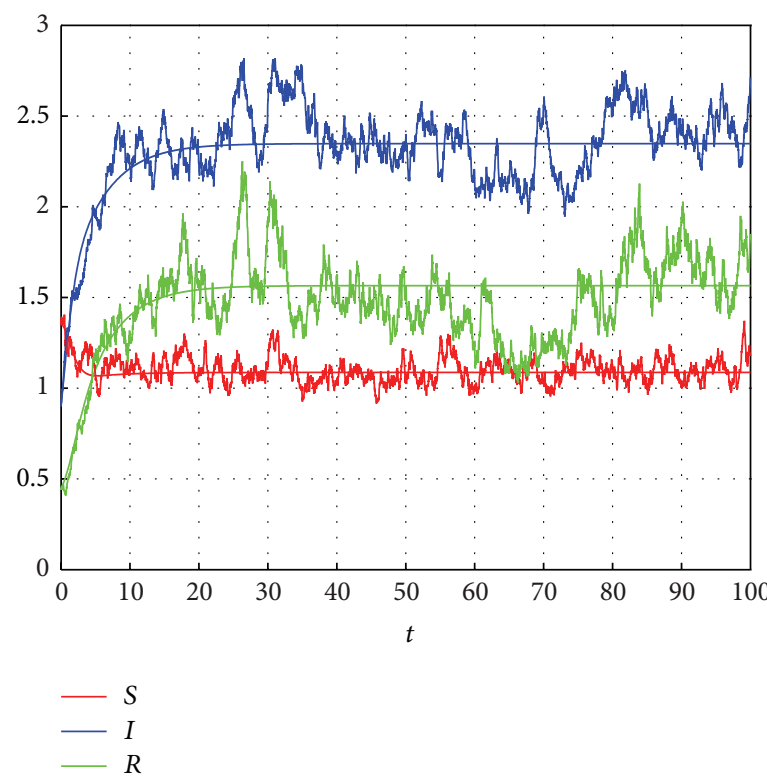

(b)

Figure 1: Solutions of model (3) with different noise. Other parameters and initial condition are given in text. (a) $\sigma_{1}=0.05, \sigma_{2}=0.01$, and $\sigma_{3}=0.03$ and $(\mathrm{b}) \sigma_{1}=0.1, \sigma_{2}=0.06$, and $\sigma_{3}=0.12$.

Considering Chebyshev inequality, Theorems 6 and 8, we immediately obtain the following result.

Theorem 9. Assume $\max \left\{\sigma_{1}^{2}, \sigma_{2}^{2}, \sigma_{3}^{2}\right\}<2(b-d)$; then the solutions of model (3) are stochastically permanent.

Proof. From Theorem 6, we have $\mathbf{P}\{|X(t)|>\delta\} \leq \varepsilon$ which implies

$$
\mathbf{P}\{|X(t)| \leq \delta\} \geq 1-\varepsilon
$$

This follows that

$$
\liminf _{t \rightarrow \infty} \mathbf{P}\{|X(t)| \leq \delta\} \geq 1-\varepsilon .
$$

By Theorem 8, we get

$$
\limsup _{t \rightarrow \infty} \mathbf{E}\left(\frac{1}{|X(t)|^{9}}\right) \leq Q .
$$

For any $\varepsilon>0$, let $\chi=\varepsilon^{9} / Q^{9}$; then

$$
\mathbf{P}\{|X(t)|<\chi\}=\mathbf{P}\left\{\frac{1}{|X(t)|}>\frac{1}{\chi}\right\} \leq \chi^{1 / 9} \mathbf{E}\left(|X(t)|^{-9}\right) .
$$

Hence,

$$
\limsup _{t \rightarrow \infty} \mathbf{P}\{|X(t)|<\chi\} \leq \chi^{1 / 9} Q=\varepsilon,
$$

which follows that

$$
\liminf _{t \rightarrow \infty} \mathbf{P}\{|X(t)| \geq \chi\} \geq 1-\varepsilon .
$$

The proof is complete.

\section{Conclusions}

In this paper, we propose an SIR epidemic model with a nonlinear incidence rate of the form $k S I /(1+\alpha I)$. We extend to consider and analyze the epidemic model with stochastic perturbations. The value of this study lies in two aspects. First, it presents existence and global stability analysis of the endemic equilibrium for the deterministic model (4). Second, it verifies some relevant properties of the corresponding stochastic model (3) and reveals the effect of environmental noise on the epidemic model.

To study the effect of environmental noise on the deterministic model (4), we stochastically perturb model (4) with respect to white noise around its endemic equilibrium. By constructing suitable Lyapunov functions and applying Itôs formula, we obtain that there is a unique positive solution to model (3) for any positive initial value and derive that the solution is stochastically bounded and permanent under some conditions. These conditions depend on the intensities of noise $\sigma_{1}, \sigma_{2}$, and $\sigma_{3}$. When the intensities of noise satisfy some conditions and are not sufficiently large, the population of the stochastic model may be stochastically permanent.

As an example, we perform some numerical simulations to illustrate the analytical results of stochastic model (3) by referring to the method mentioned in Higham [34]. Then model (3) can be rewritten as the following discretization equations:

$$
\begin{aligned}
S_{i+1}= & S_{i}+\left(b-d S_{i}-\frac{k S_{i} I_{i}}{1+\alpha I_{i}}+\gamma R_{i}\right) \Delta t \\
& +\sigma_{1}\left(S_{i}-S^{*}\right) \sqrt{\Delta t} \xi_{i}+\frac{\sigma_{1}^{2}}{2}\left(S_{i}-S^{*}\right)^{2}\left(\xi_{i}^{2}-1\right) \Delta t,
\end{aligned}
$$



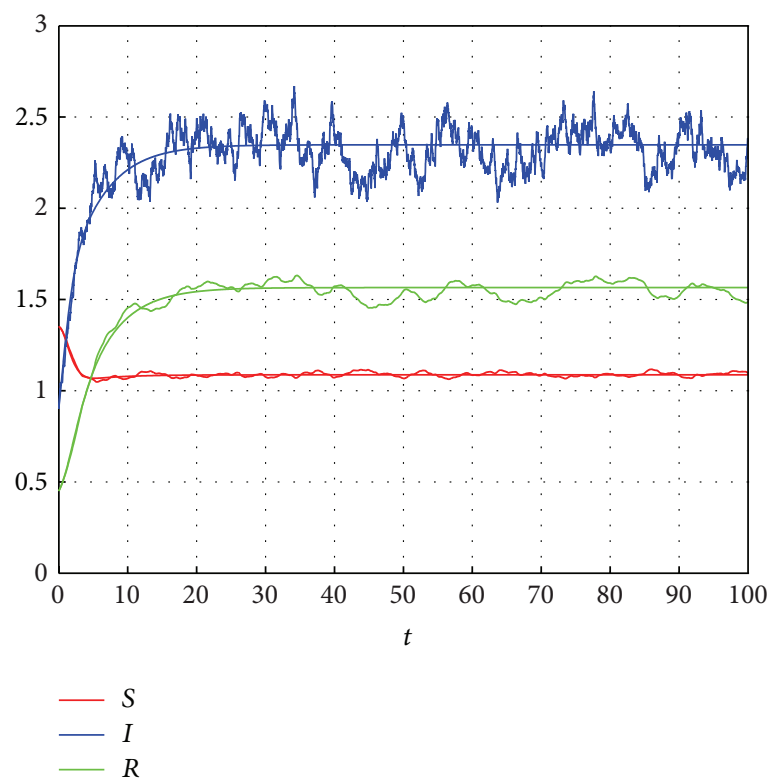
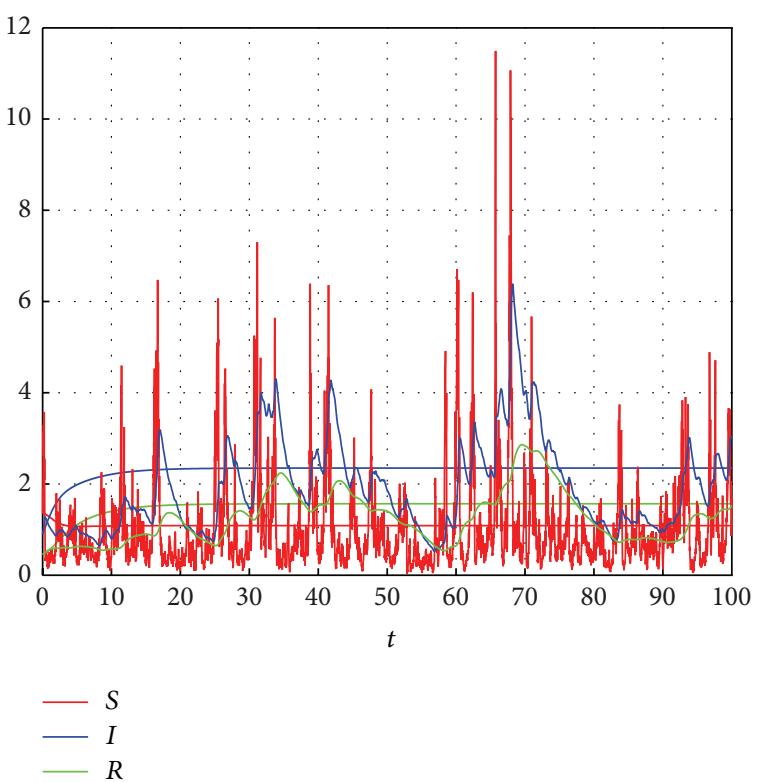

(a)

(b)

Figure 2: Solutions of model (3) with different noise. Other parameters and initial condition are the same as Figure 1 . (a) $\sigma_{1}=0, \sigma_{2}=0.06$, and $\sigma_{3}=0$ and (b) $\sigma_{1}=1.7$ and $\sigma_{2}=\sigma_{3}=0$.

$$
\begin{aligned}
I_{i+1}= & I_{i}+\left(\frac{k S_{i} I_{i}}{1+\alpha I_{i}}-(d+\mu) I_{i}\right)+\sigma_{2}\left(I_{i}-I^{*}\right) \sqrt{\Delta t} \xi_{i} \\
& +\frac{\sigma_{2}^{2}}{2}\left(I_{i}-I^{*}\right)^{2}\left(\xi_{i}^{2}-1\right) \Delta t \\
R_{i+1}= & R_{i}+\left(\mu I_{i}-(d+\gamma) R_{i}\right) \Delta t+\sigma_{3}\left(R_{i}-R^{*}\right) \sqrt{\Delta t} \xi_{i} \\
& +\frac{\sigma_{3}^{2}}{2}\left(R_{i}-R^{*}\right)^{2}\left(\xi_{i}^{2}-1\right) \Delta t
\end{aligned}
$$

where $\xi_{i}(i=1,2, \ldots, n)$ is the Gaussian random variables $N(0,1)$.

Figure 1 shows time-series plots for model (3) with and without stochastic perturbations. The parameters are taken as $b=1, d=0.2, k=1, \alpha=0.5, \gamma=$ 0.25 , and $\mu=0.3$ and initial value $(S(0), I(0), R(0))=$ $(1.35,0.9,0.45)$. In this case, model (4) has the endemic point $E^{*}=(1.087,2.3478,1.5652)$. The only difference between conditions of Figures 1(a) and 1(b) is that the values of environmental noise intensities $\sigma_{1}, \sigma_{2}$, and $\sigma_{3}$ are different. In Figure $1(\mathrm{a})$, with $\sigma_{1}=0.05, \sigma_{2}=0.01$, and $\sigma_{3}=0.03$ and in Figure 1(b), with $\sigma_{1}=0.1, \sigma_{2}=0.06$, and $\sigma_{3}=0.12$, the condition of Theorem 9 is satisfied. That is, the solutions of model (3) are stochastically permanent. From Figures 1(a) and $1(\mathrm{~b})$, one can see that with increasing the noise intensities, the solutions of model (3) will be oscillating strongly around the endemic point $E^{*}$ of model (4).

To study the effect of noise in model (3) further, in Figure 2(a), we choose $\sigma_{1}=\sigma_{3}=0, \sigma_{2}=0.06$ which satisfies the condition of Theorem 9, while in Figure 2(b), $\sigma_{1}=1.7, \sigma_{2}=\sigma_{3}=0$ that does not satisfy the condition of

Theorem 9. From Figure 2(a), one can see that the infective population $I$ will be oscillating slightly around $I^{*}=2.3478$, and both the susceptible $S$ and the removed $R$ population will be affected by the noise but the effect is very small. From Figure 2(b), when the condition of Theorem 9 is not satisfied, the noise can force the population to become largely fluctuating. In this case, the solution of model (3) is not stochastically permanent.

\section{Conflict of Interests}

The author declares that there is no conflict of interests regarding the publication of this paper.

\section{Acknowledgments}

The author thanks the editor and the anonymous referees for very helpful suggestions and comments which led to the improvement of the original paper.

\section{References}

[1] W. O. Kermack and A. G. McKendrick, "Contribution to mathematical theory of epidemics," Proceedings of the Royal Society of London A, vol. 115, no. 5, pp. 700-721, 1927.

[2] A. Abta, A. Kaddar, and H. T. Alaoui, "Global stability for delay SIR and SEIR epidemic models with saturated incidence rates," Electronic Journal of Differential Equations, vol. 2012, no. 23, pp. 1-13, 2012.

[3] E. Beretta and Y. Takeuchi, "Global stability of an SIR epidemic model with time delays," Journal of Mathematical Biology, vol. 33, no. 3, pp. 250-260, 1995. 
[4] Y. Kang and C. Castillo-Chavez, "A simple epidemiological model for populations in the wild with Allee effects and diseasemodified fitness," Discrete and Continuous Dynamical Systems $B$, vol. 19, no. 1, pp. 89-130, 2014.

[5] M. Liu and Y. H. Xiao, "Modeling and analysis of epidemic diffusion within small-world network," Journal of Applied Mathematics, vol. 2012, Article ID 841531, 14 pages, 2012.

[6] Y. Nakata, Y. Enatsu, and Y. Muroya, "On the global stability of an SIRS epidemic model with distributed delays," Discrete and Continuous Dynamical Systems A, vol. 2011, pp. 1119-1128, 2011.

[7] G. Ujjainkar, V. K. Gupta, B. Singh, R. Khandelwal, and N. Trivedi, "An epidemic model with modified non-monotonic incidence rate under treatment," Applied Mathematical Sciences, vol. 6, no. 21-24, pp. 1159-1171, 2012.

[8] D. M. Xiao and S. G. Ruan, "Global analysis of an epidemic model with nonmonotone incidence rate," Mathematical Biosciences, vol. 208, no. 2, pp. 419-429, 2007.

[9] F.-F. Zhang, G. Huo, Q.-X. Liu, G.-Q. Sun, and Z. Jin, "Existence of travelling waves in nonlinear SI epidemic models," Journal of Biological Systems, vol. 17, no. 4, pp. 643-657, 2009.

[10] J.-Z. Zhang, Z. Jin, Q.-X. Liu, and Z.-Y. Zhang, "Analysis of a delayed SIR model with nonlinear incidence rate," Discrete Dynamics in Nature and Society, vol. 2008, Article ID 636153, 16 pages, 2008.

[11] T. Zhang and Z. D. Teng, "Permanence and extinction for a nonautonomous SIRS epidemic model with time delay," Applied Mathematical Modelling, vol. 33, no. 2, pp. 1058-1071, 2009.

[12] H. El Maroufy, A. Lahrouz, and P. G. L. Leach, "Qualitative behaviour of a model of an SIRS epidemic: stability and permanence," Applied Mathematics \& Information Sciences, vol. 5, no. 2, pp. 220-238, 2011.

[13] Y. L. Cai, X. X. Wang, W. M. Wang, and M. Zhao, "Stochastic dynamics of an SIRS epidemic model with ratio-dependent incidence rate," Abstract and Applied Analysis, vol. 2013, Article ID 172631, 11 pages, 2013.

[14] A. Kaddar, "On the dynamics of a delayed SIR epidemic model with a modified saturated incidence rate," Electronic Journal of Differential Equations, vol. 2009, no. 133, pp. 1-7, 2009.

[15] A. Kaddar, "Stability analysis in a delayed SIR epidemic model with a saturated incidence rate," Nonlinear Analysis: Modelling and Control, vol. 15, no. 3, pp. 299-306, 2010.

[16] S. Pathak, A. Maiti, and G. P. Samanta, "Rich dynamics of an SIR epidemic model," Nonlinear Analysis: Modelling and Control, vol. 15, no. 1, pp. 71-81, 2010.

[17] F. A. Rihan and M. N. Anwar, "Qualitative analysis of delayed SIR epidemic model with a saturated incidence rate," International Journal of Differential Equations, vol. 2012, Article ID 408637, 13 pages, 2012.

[18] A. Korobeinikov and P. K. Maini, "Non-linear incidence and stability of infectious disease models," Mathematical Medicine and Biology, vol. 22, no. 2, pp. 113-128, 2005.

[19] V. Capasso and G. Serio, "A generalization of the KermackMcKendrick deterministic epidemic model," Mathematical Biosciences, vol. 42, no. 1-2, pp. 43-61, 1978.

[20] T. C. Gard, "Persistence in stochastic food web models," Bulletin of Mathematical Biology, vol. 46, no. 3, pp. 357-370, 1984.

[21] T. C. Gard, "Stability for multispecies population models in random environments," Nonlinear Analysis: Theory, Methods \& Applications, vol. 10, no. 12, pp. 1411-1419, 1986.

[22] P. E. Greenwood and L. F. Gordillo, "Stochastic epidemic modeling," in Mathematical and Statistical Estimation Approaches in Epidemiology, pp. 31-52, Springer, Amsterdam, The Netherlands, 2009.

[23] Y. Kang and D. Armbruster, "Noise and seasonal effects on the dynamics of plant-herbivore models with monotonic plant growth functions," International Journal of Biomathematics, vol. 4, no. 3, pp. 255-274, 2011.

[24] F. Rao, "Dynamical analysis of a stochastic predator-prey model with an Allee effect," Abstract and Applied Analysis, vol. 2013, Article ID 340980, 10 pages, 2013.

[25] F. Rao, "The complex dynamics of a stochastic toxicphytoplankton-zooplankton model," Advances in Difference Equations, vol. 2014, no. 1, article 22, 2014.

[26] F. Rao, S. J. Jiang, Y. Q. Li, and H. Liu, "Stochastic analysis of a Hassell-Varley type predation model," Abstract and Applied Analysis, vol. 2013, Article ID 738342, 10 pages, 2013.

[27] F. Rao, W. M. Wang, and Z. B. Li, "Stability analysis of an epidemic model with diffusion and stochastic perturbation," Communications in Nonlinear Science and Numerical Simulation, vol. 17, no. 6, pp. 2551-2563, 2012.

[28] H. C. Tuckwell and R. J. Williams, "Some properties of a simple stochastic epidemic model of SIR type," Mathematical Biosciences, vol. 208, no. 1, pp. 76-97, 2007.

[29] L. Y. Wang, H. L. Huang, A. C. Xu, and W. M. Wang, "Stochastic extinction in an SIRS epidemic model incorporating media coverage," Abstract and Applied Analysis, vol. 2013, Article ID 891765, 8 pages, 2013.

[30] X. X. Wang, H. L. Huang, Y. L. Cai, and W. M. Wang, "The complex dynamics of a stochastic predator-prey model," Abstract and Applied Analysis, vol. 2012, Article ID 401031, 24 pages, 2012.

[31] E. Beretta, V. Kolmanovskii, and L. Shaikhet, "Stability of epidemic model with time delays influenced by stochastic perturbations," Mathematics and Computers in Simulation, vol. 45, no. 3-4, pp. 269-277, 1998.

[32] A. Bahar and X. Mao, "Stochastic delay Lotka-Volterra model," Journal of Mathematical Analysis and Applications, vol. 292, no. 2, pp. 364-380, 2004.

[33] D. Q. Jiang, N. Shi, and X. Y. Li, "Global stability and stochastic permanence of a non-autonomous logistic equation with random perturbation," Journal of Mathematical Analysis and Applications, vol. 340, no. 1, pp. 588-597, 2008.

[34] D. J. Higham, "An algorithmic introduction to numerical simulation of stochastic differential equations," SIAM Review, vol. 43 , no. 3, pp. 525-546, 2001. 


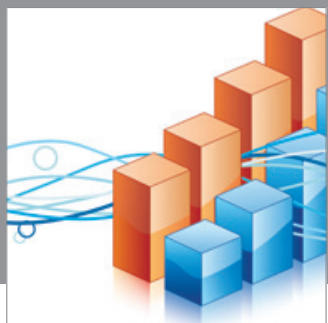

Advances in

Operations Research

mansans

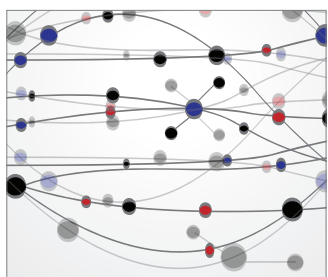

The Scientific World Journal
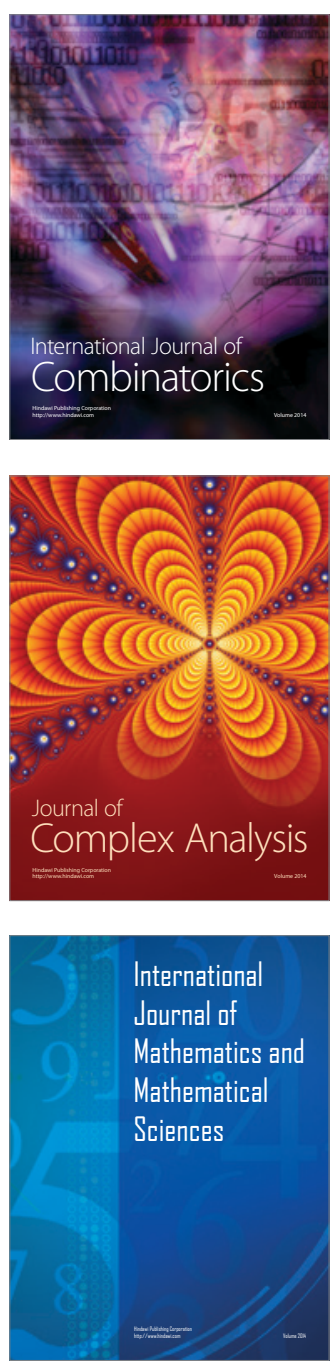
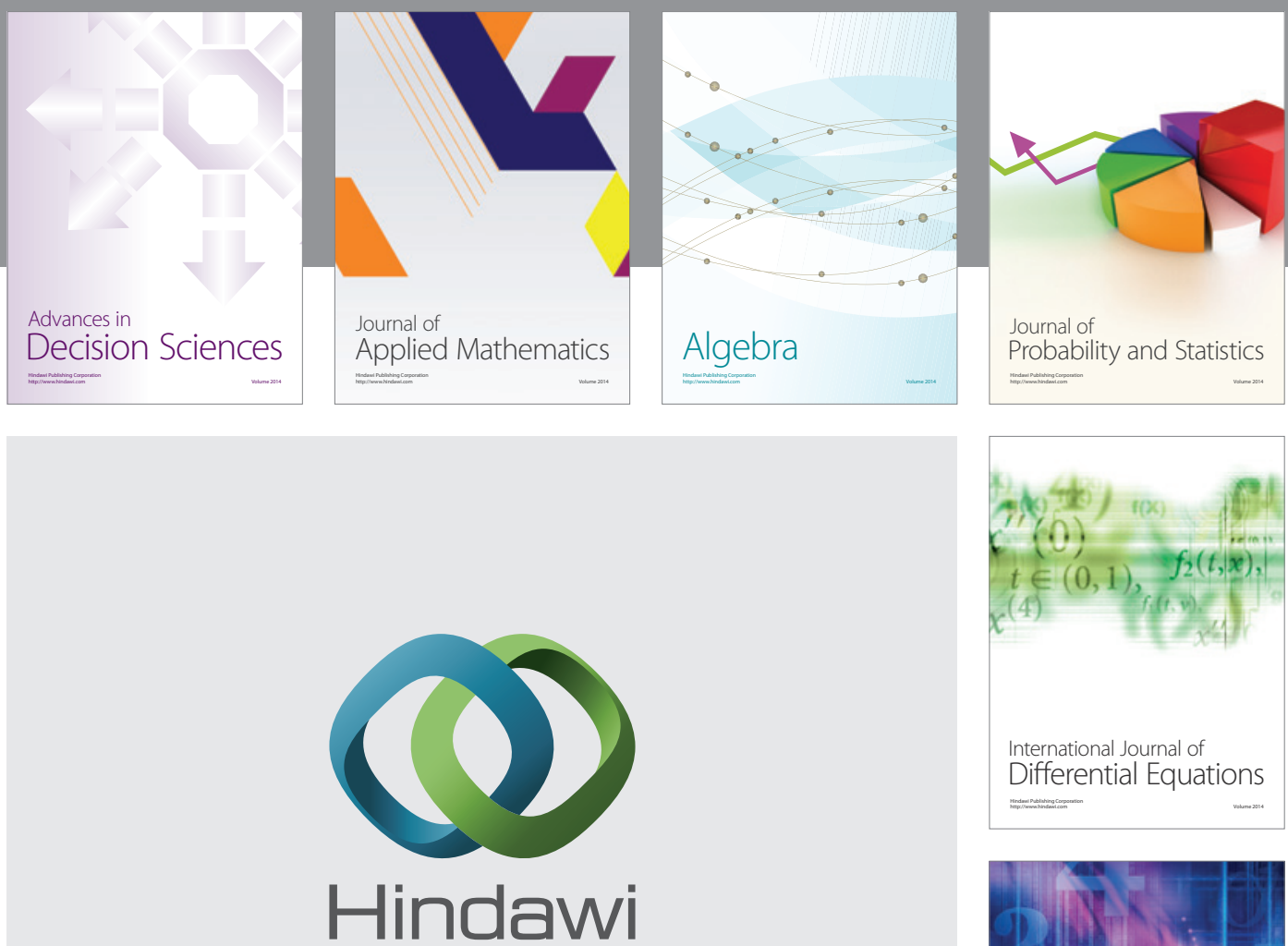

Submit your manuscripts at http://www.hindawi.com
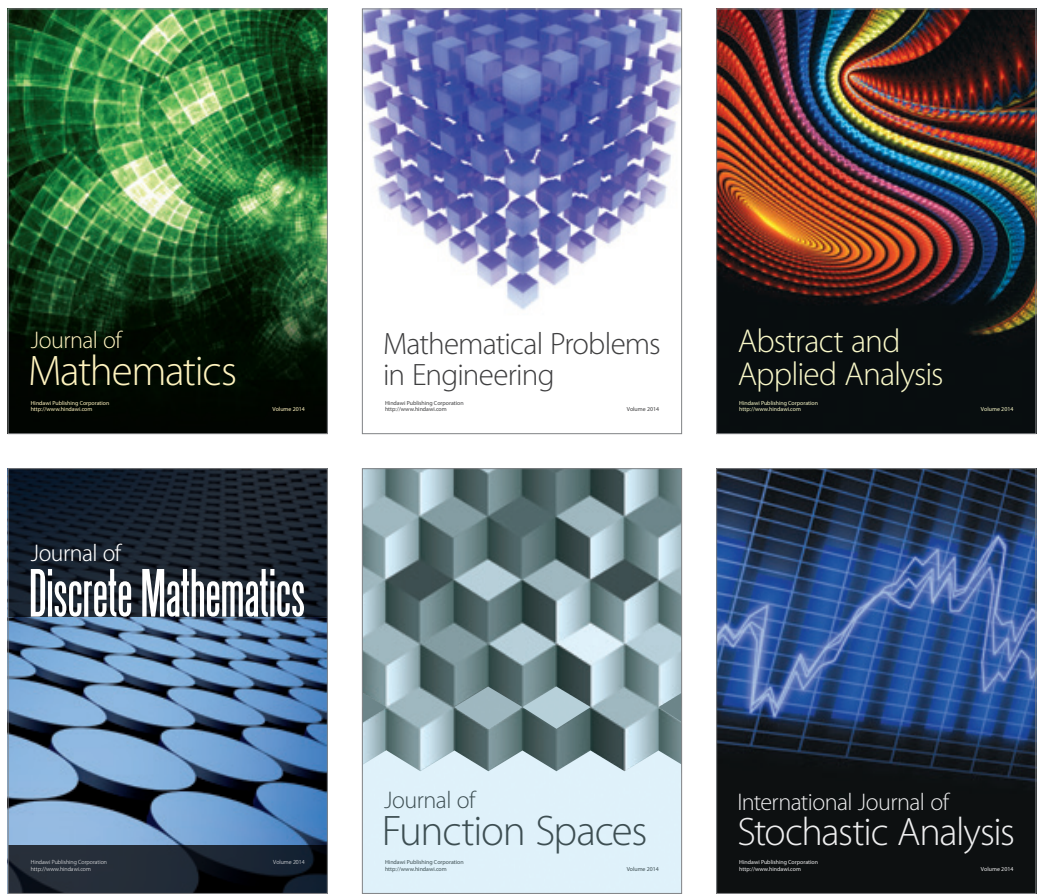

Journal of

Function Spaces

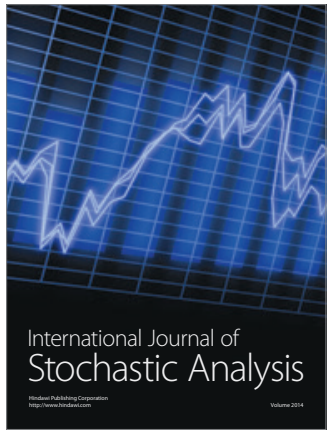

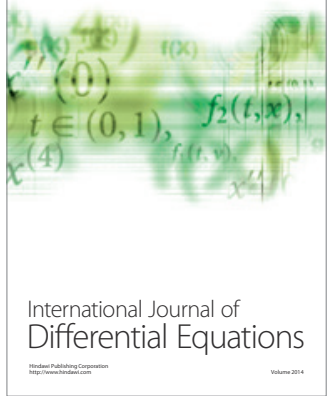
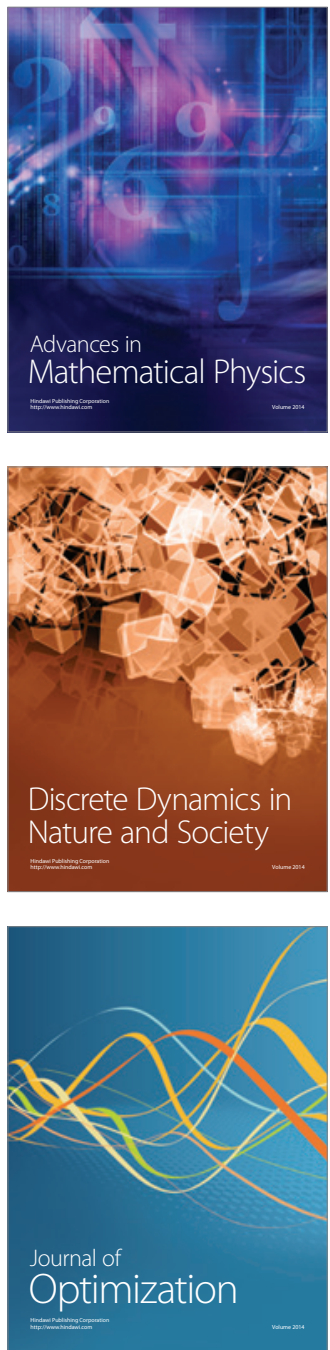